\title{
ON THE RELATION BETWEEN POWER INPUT AND IMPELLER JET FLOW RATE
}

\author{
SETSURO HIRAOKA* AND RYUZo ITO ${ }^{1}$ \\ Department of Industrial Chemistry \\ Nagoya Institute of Technology, Nagoya, 466
}

\begin{abstract}
Based on the flow pattern in impeller region and on the angular momentum balance over the potential flow region, a relationship between impeller jet flow rate and power input is derived in which the impeller jet flow rate is proportional to the root of power input. The correlation equations based on this result are well in agreement with the experimental results for baffled and non-baffled agitated vessels with different type impellers.
\end{abstract}

\section{Introduction}

An agitated vessel has often been used as a mixing device in chemical processes, and the mixing performance in the vessel directly depends on the impeller jet flow rate. The impeller jet flow rate has been studied by many workers and correlated with the power input based on dimensional analysis. However, due to the complexity of flow pattern in an agitated vessel, no useful correlation for jet flow rate has yet been obtained. Recently Nagase et al. ${ }^{6}$ have obtained a new correlation of circulation flow rate and power input, based on the vorticity balance and dimensional analysis.

This paper deals with the derivation of correlation between power input and impeller jet flow rate from the angular momentum balance and flow pattern for the turbulent region, and will discuss the applicability of the present correlations.

\section{Analysis}

Assuming that fluid is incompressible and that tangential flow is axisymmetrical, the equation of motion for the tangential component and the equation of continuity respectively are reduced to

$$
\begin{gathered}
\rho\left\{u \frac{\partial(r w)}{\partial r}+v \frac{\partial(r w)}{\partial z}\right\}=-\frac{1}{r}\left\{\frac{\partial\left(r^{2} \tau_{r \theta}\right)}{\partial r}+\frac{\partial\left(r^{2} \tau_{z \theta}\right)}{\partial z}\right\} \\
\frac{\partial(r u)}{\partial r}+\frac{\partial(r v)}{\partial z}=0
\end{gathered}
$$

By using Eq. (2), Eq. (1) can be rearranged as follows:

$$
\rho\left\{\frac{\partial\left(r^{2} u w\right)}{\partial r}+\frac{\partial\left(r^{2} v w\right)}{\partial z}\right\}=-\left\{\frac{\partial\left(r^{2} \tau_{r \theta}\right)}{\partial r}+\frac{\partial\left(r^{2} \tau_{z \theta}\right)}{\partial z}\right\}
$$

Received September 27, 1974.

Presented at the 8th Autumn Meeting of The Soc. of Chem. Engrs., Japan, at Tokyo, Oct. 1974.

1 Dept. of Chem. Eng., Faculty of Engineering Science, Osaka Univ., Toyonaka, 560.
For the potential flow region $\left(r_{d} \leqq r \leqq r_{D}, 0 \leqq z \leqq H\right)$ in an agitated vessel without free surface, the following boundary conditions hold, because the viscous shear stress at $r_{d}$ can be neglected under turbulent flow conditions.

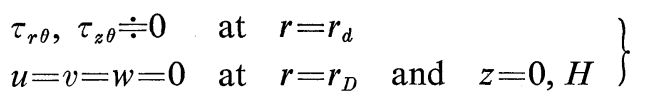

Based on these conditions, Eq. (3) is averaged with respect to time, and integrated with respect to $r$ and $z$ as follows:

$$
\begin{aligned}
& \rho\left\{\int_{0}^{H}\left(\left.r^{2} U W\right|_{r_{d}}\right) d z+\int_{0}^{H}\left(r^{2} \overline{\left.u^{\prime} w^{\prime}\right|_{r_{d}}}\right) d z\right\} \\
= & \int_{0}^{H}\left(\left.r^{2} \bar{\tau}_{r_{\theta}}\right|_{r_{D}}\right) d z+\int_{r_{d}}^{r_{D}} r^{2}\left(\left.\bar{\tau}_{z \theta}\right|_{H}-\left.\bar{\tau}_{z \theta}\right|_{0}\right) d r
\end{aligned}
$$

where the capitals express the time-averaged velocities and $\rho \overline{u^{\prime} w^{\prime}}$ is Reynolds stress component.

It is known experimentally that the first term on both sides of Eq. (5) is much greater than the second to the same extent, so that the latter on both sides may be neglected simultaneously without large error ${ }^{5}$.

$$
\rho \int_{0}^{H}\left(\left.r^{2} U W\right|_{r_{d}}\right) d z \div \int_{0}^{H}\left(\left.r^{2} \tilde{\tau}_{\boldsymbol{r} \theta}\right|_{r_{D}}\right) d z
$$

Multiplying Eq. (6) into $2 \pi$, the right-hand side is equal to the torque at the side wall of the vessel, and the left-hand side is equal to the angular momentum transferred by the impeller jet flow from the impeller tip to the potential flow region.

For the fully turbulent agitated vessel, it is well known that the tangential velocity in potential flow region satisfies the relation of $r W \doteqdot$ const. except the height being impeller. Then the tangential velocity at the suction point, where $r=r_{d}$ and $\left.U\right|_{r_{d}}<0$, is assumed as $\left.W\right|_{r_{d}} \equiv W_{d} \doteqdot$ const. The tangential velocity of fluid at the injection point, where $r=r_{d}$ and $\left.U\right|_{r_{d}}$ $<0$, is assumed to equal that at the impeller tip, i.e. $\left.W\right|_{r_{d}}=W_{0}$. 


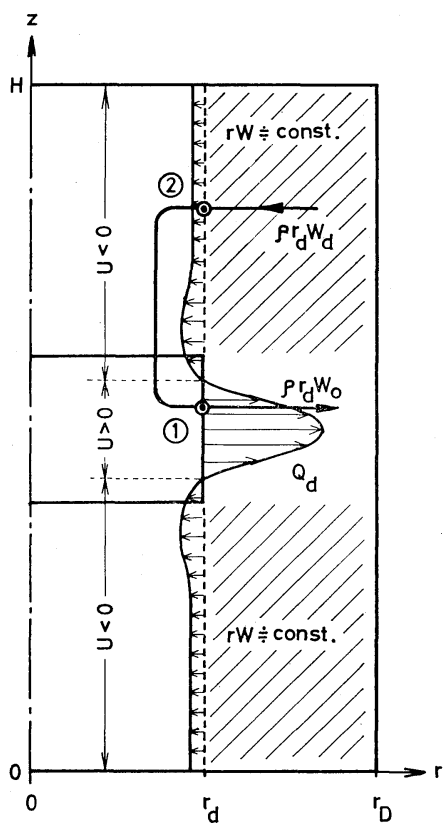

Fig. 1 Schematic diagram of impeller jet flow and angular momentum balance

Table 1 Comparison between experimental and calculated values of impeller jet flow rate for the vessel without free surface

\begin{tabular}{cccrcc} 
No. & $d / D$ & $b / D$ & $n_{p}$ & $\left(N_{q_{d}}\right)_{e x p}$ & $\left(N_{q_{d}}\right)_{c a l}$ \\
\hline 1 & 0.61 & 0.10 & 8 & 0.12 & 0.12 \\
2 & 0.50 & 0.10 & 8 & 0.18 & 0.21 \\
3 & 0.48 & 0.20 & 8 & 0.24 & 0.23 \\
4 & 0.47 & 0.30 & 8 & 0.26 & 0.25 \\
\hline
\end{tabular}

Based on these assumptions, both sides of Eq. (6) multiplied into $2 \pi$ can be expressed as

$$
\begin{aligned}
2 \pi \int_{0}^{H}\left(\left.r^{2} \bar{\tau}_{r \theta}\right|_{r_{D}}\right) d z & =\frac{\pi}{2} D^{2} H \cdot \bar{\tau}_{w} \\
2 \pi \rho \int_{0}^{H}\left(\left.r^{2} U W\right|_{r_{d}}\right) d z & =2 \pi \rho r_{d}^{2}\left\{W_{0} \int_{U>0}\left(\left.U\right|_{r_{d}}\right) d z\right. \\
\left.+W_{d} \int_{U<0}\left(\left.U\right|_{r_{d}}\right) d z\right\} & =\rho r_{d}\left(W_{0}-W_{d}\right) Q_{d}
\end{aligned}
$$

where $Q_{d}$ is the impeller jet flow rate, defined as

$$
Q_{d}=2 \pi r_{d} \int_{U>0}\left(\left.U\right|_{r_{d}}\right) d z=-2 \pi r_{d} \int_{U<0}\left(\left.U\right|_{r_{d}}\right) d z
$$

In the non-baffled fully turbulent agitated vessel with paddle impeller, the tangential velocity at the suction point $W_{d}$ has been confirmed experimentally to satisfy the following relation ${ }^{3)}$.

$$
\left(W_{0}-W_{d}\right) / \bar{u}_{w}^{*}=10
$$

Considering that $\bar{u}_{w}^{*}$ is equal to $\sqrt{\bar{\tau}_{w} / \rho}$, the combination of Eqs. (6), (7) and (9) gives a simple relationship between impeller jet flow rate and friction velocity.

$$
Q_{d}=(\pi / 10)\left(D^{2} H / d\right) \bar{u}_{w}^{*}
$$

By using the dimensionless groups $N_{P}$ and $N_{q_{d}}$, Eq. (10) is easily rearranged to become

$$
N_{q_{d}}=\frac{1}{10} \sqrt{\left(\frac{D^{2} H}{d^{3}}\right) \cdot \frac{N_{P}}{1+\alpha}}
$$

where

$$
\left.\begin{array}{l}
N_{P}=\frac{\pi^{2}(1+\alpha) D^{2} H \bar{\tau}_{w}}{\rho N^{2} d^{5}}=\frac{\pi^{2}(1+\alpha) D^{2} H}{N^{2} d^{5}}\left(\bar{u}_{w}^{*}\right)^{2} \\
N_{q_{d}}=\frac{Q_{d}}{N d^{3}}
\end{array}\right\}
$$

Equation (11) shows that the impeller jet flow rate is proportional to the square root of power input. This result is similar to that of Nagase, i.e., the circulation flow rate is proportional to the square root of power input ${ }^{6}$.

$$
\frac{N_{q_{c}}}{2}=\frac{1}{\sqrt{ } 12.2}\left(\frac{D}{d}\right) \sqrt{\frac{N_{P}}{2}}
$$

\section{Experimental Results and Discussion}

\section{Vessel without free surface}

The three dimensional velocity profiles in an agitated vessel without free surface have been measured for four-paddle impellers ${ }^{5}$. The values of $N_{q_{d}}$ are obtained through the graphical integration of impeller jet flow pattern and are expressed in Table 1. The experimental results are in good agreement with the values calculated by Eq. (11), where the power input is given by the following equation ${ }^{2,4}$.

$$
\frac{f}{2}=\frac{\tilde{\tau}_{w}}{\rho v_{\theta}^{2}}=0.121\left(\frac{L v_{\theta} \rho}{\mu}\right)^{-1 / 3}
$$

\section{Vessel with free surface}

It is examined whether Eq. (11) derived for the vessel without free surface is applicable to the vessel with free surface.

Three-dimensional velocity profiles in an agitated vessel with free surface have been measured by Yamamoto $^{8)}$ and Nagase et al. ${ }^{91}$. The tangential velocity at the suction point $W_{d}$ in these results can be replotted to correlate as shown in Fig. 2, where the abscissa $\gamma\left(n_{p} b / D\right)$ is the similarity condition parameter ${ }^{4}$. The calculated values of $\bar{u}_{w}^{*}$ are used, because the experimental values of power input at these flow conditions are scarcely known. Here $\bar{u}_{w}^{*}$ is derived from the definition, by calculating $\bar{\tau}_{w}$ with Eq. (14) and multiplying it by two, sed on the fact that the power inputs calculated by 1. (14) are about half of the experimentally obtaine values of that for the vessel with free surface ${ }^{8)}$.

In Fig. 2 the ordinate seems to be proportional to the one-third power of similarity condition parameter, in contrast to Eq. (9) for the vessel without free surface. The correlation equation is shown as

$$
\frac{\left(W_{0}-W_{d}\right)}{\bar{u}_{w}^{*}}=6.2\left(\cdot \frac{n_{p} b}{D}\right)^{-1 / 3}
$$

Assuming that Eqs. (6) and (7) hold for the vessel with free surface, the combination of Eqs. (6), (7) and 
(15) gives the following correlation equation for the vessel with free surface in the same manner as Eq. (11).

$$
N_{q_{d}}=0.16\left(\gamma \cdot \frac{n_{p} b}{D}\right)^{1 / 3} \sqrt{\left(\frac{D^{2} H}{d^{3}}\right) \frac{N_{P}}{1+\alpha}}
$$

The impeller jet flow rate values obtained by Yamamoto $^{8)}$ and Nagase et al. ${ }^{9)}$ are compared with the values calculated by Eq. (16) as shown in Fig. 3, where $\alpha$ is assumed to be $0.2^{1)}$. The experimental data represent partly the baffled agitated vessel and partly the different type impellers. For a wide range of $N_{q_{d}}$ most data satisfy the functional relationship between $N_{P}$ and $N_{q_{d}}$ of Eq. (16) obtained for the non-baffled agitated vessel with paddle impeller, but the experimental value is about 1.6 times greater than the calculated one. Then the empirical equation is modified as

$$
N_{q_{d}}=0.26\left(\gamma \cdot \frac{n_{p} b}{D}\right)^{1 / 3} \sqrt{\left(\frac{D^{2} H}{d^{3}}\right) \frac{N_{P}}{1+\alpha}}
$$

\section{Relation of Impeller Jet Flow and Circulation Flow}

The relationship between impeller jet flow rate and circulation flow rate can be easily derived from Eqs. (13) and (16a) for the vessel with free surface.

$$
N_{q_{c}}=0.78 \sqrt{1+\alpha}\left(\gamma \frac{n_{P} b}{D}\right)^{-1 / 3} \sqrt{\frac{d}{H}} N_{q_{d}}
$$

On the other hand, Nagata et $a .^{7)}$ have proposed on empirical equation for the circulation flow rate:

$$
N_{q_{c}}=\left[1+0.16\left\{(D / d)^{2}-1\right\} N_{q_{d}}\right.
$$

As the vessel diameter equals the vessel height, the ratio of $N_{q_{c}}$ and $N_{q_{d}}$ decreases with decreasing $d / D$ from Eq. (17), but on the contrary increases from Eq. (18). Thus, it is impossible at present to concluded which of Eq. (17) and (18) should best be used for design purposes.

\section{Conclusive Remark}

Based on the flow pattern in impeller region and on the angular momentum balance over the potential flow region, the relationship between impeller jet flow rate and power input is derived and compared with the experimental results. The correlation equations are in good agreement with the experimental results for baffled and nonbaffled agitated vessels with different type impellers.

\section{Nomenclature}

$b \quad=$ impeller width

d $\quad=$ impeller diameter

D $\quad=$ vessel diameter

$f \quad=$ friction factor

$H \quad=$ vessel height

$L=$ characteristic length $(=(D / 2) \ln (D / d))$

$N \quad=$ rotational speed

$n_{p} \quad=$ impeller blade number

$P \quad=$ power input

[cm]

[cm]

[cm]

[-]

[cm] $\left[\mathrm{g} \cdot \mathrm{cm}^{1} / \mathrm{sec}^{3}\right]$

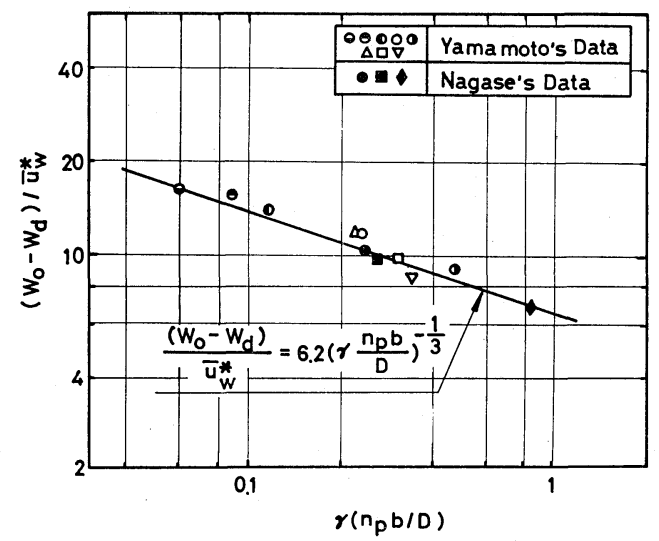

Fig. 2 Correlation of the tangential velocity at the suction point with the similarity condition parameter for the vessel with free surface

\begin{tabular}{|c|c|c|}
\hline$Q_{c}$ & $=$ circulation flow rate & {$\left[\mathrm{cm}^{3} / \mathrm{sec}\right]$} \\
\hline$Q_{d}$ & $=$ impeller jet flow rate & {$\left[\mathrm{cm}^{3} / \mathrm{sec}\right]$} \\
\hline & $=$ radius & \\
\hline$r_{d}$ & $=$ impeller radius $(=d / 2)$ & \\
\hline$r_{D}$ & $=$ vessel radius $(=D / 2)$ & \\
\hline$U$ & $=$ time averaged radial velocity & {$[\mathrm{cm} / \mathrm{s}$} \\
\hline$u$ & $=$ radial velocity & {$[\mathrm{cm} / \mathrm{s}$} \\
\hline $\bar{u}_{w}^{*}$ & $=$ friction velocity $\left(=\sqrt{\overline{\bar{\tau}_{w}} / \rho}\right)$ & {$[\mathrm{cm} / \mathrm{se}$} \\
\hline$\rho \overline{u^{\prime} w^{\prime}}$ & $=$ Reynolds stress component & $/ \mathrm{cm} \cdot \mathrm{sec}$ \\
\hline$v$ & $=$ axial velocity & {$[\mathrm{cm} /$} \\
\hline$v_{\theta}$ & $=$ characteristic velocity $(=(\pi / 2) N d \beta)$ & {$[\mathrm{cm} /$} \\
\hline$W$ & $=$ time averaged tangential velocity & {$[\mathrm{cm} / \mathrm{s}$} \\
\hline$W_{0}$ & $=$ impeller tip velocity $\left(=2 \pi N r_{d}\right)$ & {$[\mathrm{cm} / \mathrm{s}$} \\
\hline$W_{d}$ & $\begin{array}{l}=\text { tangential velocity at suction point } \\
\text { (' } 2 \text { ' in Fig. 1) }\end{array}$ & {$[\mathrm{cm} / \mathrm{s}$} \\
\hline$w$ & $=$ tangential velocity & {$[\mathrm{cm} / \mathrm{s}$} \\
\hline$z$ & $=$ axial direction & \\
\hline$\alpha$ & $\begin{array}{l}=\text { ratio of torque at bottom wall to that } \\
\text { at side }\end{array}$ & \\
\hline$\beta$ & $=$ variable defined by $2 \ln (D / d) /(D / d-d / L$ & \\
\hline$\gamma$ & $=$ variable defined by $\left\{(d / D)^{5} \ln (D / d) / \beta^{5}\right\}^{1 /}$ & \\
\hline$\rho$ & $=$ density & \\
\hline$\mu$ & $=$ viscosity & \\
\hline
\end{tabular}

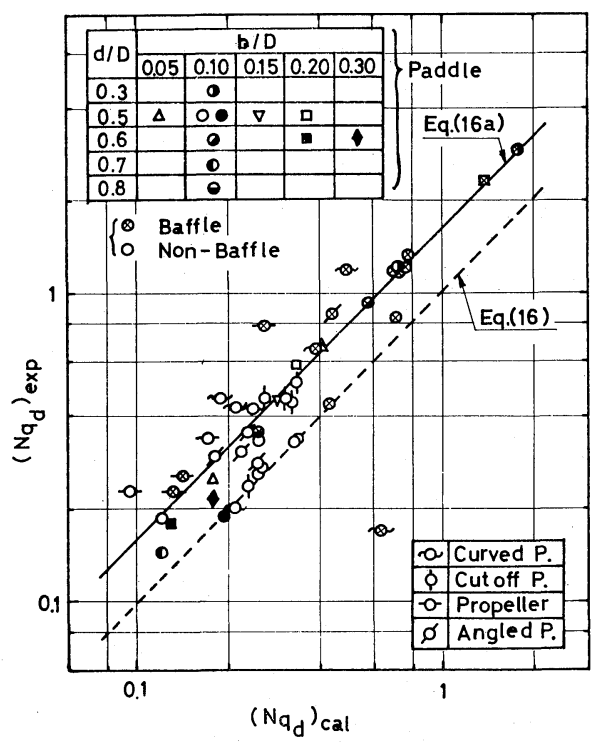

Fig. 3 Comparison between experimental and calculated values of impeller jet flow rate for the vessel with free surface 


$\begin{array}{lll}\tau_{r \theta}, \tau_{z \theta}= & \text { viscous shear stress component } & {\left[\mathrm{g} / \mathrm{cm} \cdot \mathrm{sec}^{2}\right]} \\ \bar{\tau}_{r \theta}, \bar{\tau}_{r \theta}= & \text { time averaged viscous shear stress } & \\ & \text { component } & {\left[\mathrm{g} / \mathrm{cm} \cdot \mathrm{sec}^{2}\right]} \\ \tau_{w} & =\text { shear stress at wall } & {\left[\mathrm{g} / \mathrm{cm} \cdot \mathrm{sec}^{2}\right]} \\ \bar{\tau}_{w} & =\text { average shear stress over side wall } & {\left[\mathrm{g} / \mathrm{cm} \cdot \mathrm{sec}^{2}\right]} \\ N_{p} & =P / \rho N^{3} d^{5} & \\ N_{q_{c}} & =Q_{c} / N d^{3} & \\ N_{q_{d}} & =Q_{d} / N d^{3} & \end{array}$

\section{Literature Cited}

1) Hiraoka, S., M. Hattori and I. Yamada: Kagaku Kōgaku, 37, 202 (1973).

2) Hiraoka, S. and R. Ito: J. Chem. Eng. Japan, 6, 464 (1973).

3) Hiraoka, S., R. Ito, I. Yamada, K. Sawada, M. Ishiguro and S. Kawamura: ibid., 8, 156 (1975).

4) Hiraoka, S., I. Yamada, N. Doi, H. Takeda, A. Kawai, Y. Usui and R. Ito: Bull. Nagoya Inst. of Tech., 26, 239 (1974).

5) Mizushina, T., R. Ito, S. Hiraoka and K. Fujimoto: $\mathrm{Ka}$ gaku Kögaku, 37, 409 (1973).

6) Nagase, Y., T. Iwamoto, S. Fujita and T. Yoshida: ibid., 38, 519 (1974).

7) Nagata, S., K. Yamamoto, M. Ujihara, K. Hashimoto and Y. Naruse: ibid., 23, 130, 595 (1959).

8) Yamamoto, K.: Dr. Thesis, Kyoto Univ., Kyoto, Japan (1960).

9) Yoshida, T., Y. Nagase, M. Kakumoto, T. Hasegawa and M. Matoba: Kagaku Kögaku, 37, 1038 (1973).

\title{
SOLUBILITIES OF METHANE IN METHANOL-WATER AND ETHANOL-WATER SOLUTIONS
}

\author{
JUNJI TOKUNAGA* AND MAKoto KAWAI \\ Department of Chemical Engineering, Kansai University, Suita, Osaka, 564
}

In a previous paper ${ }^{4)}$, one of the authors showed a general correlation for non-polar gas solubilities in aqueous alcohol solutions. The correlation was derived from the solubility data of oxygen, nitrogen, and carbon dioxide in aqueous solutions with methanol, ethanol, 1-propanol, or 2-propanol.

In this work, solubility measurements of methane in aqueous solutions with methanol or ethanol were made at $20^{\circ} \mathrm{C}$ and the results were compared with the general correlation.

\section{Experimental}

\section{Apparatus and Materials}

The apparatus and procedure are the same as those in the previous papers ${ }^{3}{ }^{5}$.

Methane used was pure gas obtained from Seitetsu Kagaku Co., which was certified more than $99.0 \%$ pure. Methanol and ethanol were guaranteed reagents, manufactured by Wako Pure Chemical Ind. These reagents were further purified in a laboratory fractionating column. Physical properties of the purified solvents are shown in Table 1.

\section{Experimental results}

Tables 2 and 3 show the experimental solubilities of methane in methanol (1)-water (2) and ethanol (1)-

Received October 25, 1974. water (2) solutions at $20^{\circ} \mathrm{C}$ and $760 \mathrm{mmHg}$ partial pressure of methane. Gas solubilities in pure solvents are the averages of three determinations. They are compared with the literature values in Table 4. The reproducibility of experimental solubilities was within about $1 \%$ error on the average.

\section{Discussion}

In the previous paper ${ }^{4}$, it was reported that the relationship between the excess quantities expressed by Eq. (1) and the composition of aqueous alcohol solutions was given by a particular curve for each kind of alcohol in the solutions, regardless of the kind of solute gases, and these curves were expressed by Eq. (2) for each aqueous alcohol solution. The constants in Eq. (2) were determined from the solubility data of oxygen, nitrogen, and carbon dioxide.

$$
\begin{aligned}
& \ln \kappa(L)=\ln L_{\mathrm{R}, \mathrm{mix}}-\sum_{i=1}^{2} \Phi_{i} \ln L_{\mathrm{R}, i} \\
& \ln \kappa(L)=\Phi_{1} \Phi_{2}\left(\mathrm{~A}+\mathrm{B} \Phi_{1}+\mathrm{C} \Phi_{1}^{2}+\mathrm{D} \Phi_{1}^{3}\right)
\end{aligned}
$$

Table 1 Physical properties of pure solvents used Density $d_{4}^{25} \quad$ Refractive index $n_{D}^{25}$ Obsd. Lit. Obsd. Lit.

$\begin{array}{lllll}\text { Methanol } & 0.78681 & 0.78664^{2)} & 1.32666 & 1.32652^{2)}\end{array}$

$\begin{array}{llllll}\text { Ethanol } & 0.78508 & 0.78504^{2)} & 1.35891 & 1.35941^{2)}\end{array}$

